\title{
Training Quench Performance and Quench Location of the Short Superconducting Dipole Models for the LHC
}

\author{
S. Sanfilippo, A. Siemko, D. Tommasini, and W. Venturini-Delsolaro
}

\begin{abstract}
The short model program, started in October 1995 to study and validate design variants and assembly of the main Large Hadron Collider (LHC) dipoles, has achieved its last phase. The last models were focused on the validation of specific design choices to be implemented in the series production, and to the study of the training performance of the coil heads. This paper reports on the manufacturing features of the recent twin-aperture short models, reviews the results of the cold tests and presents a summary of the training quench performance and quench location.
\end{abstract}

Index Terms-Accelerator magnets, quench location.

\section{The Model Program}

$\mathbf{F}$ OLLOWING the approval of the Large Hadron Collider Project (LHC) by the CERN Council [1], an intensive program of 1-m long dipole models started in 1995, based on the manufacture and test of single-aperture and double-aperture 1-m long models. These models allowed to select the series-design features among several variants for the coil cross section [2], the cable insulation, the material of the collars and of the coil end-spacers and the coil pre-stress [3], [4], and the relation between field harmonics and coil size [5]. The recent double-aperture models were dedicated to explore the quench performance in different manufacturing configurations continuing the work reported in [6]. This paper reports about the fabrication and testing of these magnets, and comparisons with the results obtained from selected past magnets.

\section{FeAtures of Recent Models}

The layout of the short dipole models was already presented in previous papers [7]. The double-aperture models feature the same collars and yoke laminations as the main dipoles, held together by a bolted shrinking cylinder for easy re-assembly of the structure.

Four double-aperture models have been manufactured and tested during the last year, called T8.V1, T9.V1, T10.V1\&V2, and T11.V1. The cable characteristics, the coil cross section and the material of the coil end spacers were the same in all cases.

The main specific variants implemented in these models were the layout of conductor blocks in the outer layer coil heads, the effect of using metallic innermost end spacers, the matching between magnet straight section and ends and

Manuscript received September 24, 2001.

The authors are with CERN, CH-1211 Geneva 23, Switzerland (e-mail: stephane.sanfilippo@cern.ch).

Publisher Item Identifier S 1051-8223(02)03469-3.
TABLE I

MAIN SPECIFIC FEATURES OF RECENT SHORT DiPOLES

\begin{tabular}{cl}
\hline Magnet & \multicolumn{1}{c}{ Specific features } \\
\hline T5.V3 & reference magnet. \\
T4.V4 & reference for T4.V6. \\
T4.V6 & innermost outer layer end spacers made of austenic steel. \\
T8.V1 & coils wound in "as collared" configuration. \\
T9.V1 & additional conductor block on the outer layer heads. \\
T10.V1 & as T9.V1 but different matching between straight part and ends. \\
T10.V2 & as T10.V1, but with yoke of new LHC baseline design. \\
T11.V1 & as T9.V1, but different fabrication of coil ends. \\
\hline
\end{tabular}

the fabrication process of the coil ends. These variants are summarized in Table I.

\section{DETAILS OF FABRICATION VARIANTS}

Considering T5.V3 as reference (six-block coil cross section, all end-spacers of isoperimetric shape made of fiberglass, austenitic steel collars, and other assembly details described in [6]), we express below the specific features of each of the magnets discussed in this paper.

T4.V6 was made by replacing the resin-fiberglass innermost major end spacers of the outer layer of the previous version T4.V4 with austenitic steel pieces. The cables remained the same. The aim of this variant was to check if a coil with a similar elastic modulus of the straight section (where the coil is supported by the austenitic steel collars nose) and the transition region close to the heads gave a better training performance.

T8.V1 was an attempt to build the coils in a configuration as close as possible to that of the collared magnet. In particular, the coil ends were already shimmed in the mid-plane at the end of the winding process, before the thermal bonding-sizing cycle. In this way the coil heads were supposed to become more compact and assume a geometry already similar to the one after collaring. The shims to be added in the mid-plane to obtain the correct pre-stress in the coil heads were in fact considerably smaller than usual (about $0.2-0.3 \mathrm{~mm}$ instead of about $0.5 \mathrm{~mm}$ ). However bigger shims had to be used during the coil fabrication, because before the thermal bonding-sizing cycle, the coil is softer than after it. It should be recalled that the coil ends, after the thermal bonding-sizing cycle, are in fact impregnated with resin to become more compacted and rigid. As a result, this technique of fabrication may have deformed too much the coil ends.

For the next magnets, T9.V1, T10.V1, and T11.V1, the collar geometry had changed, requiring a new study of the matching between coil straight section and heads. This produced certain 
problems for the reproducibility of short dipole models. We could consider T9.V1 and T10.V1 very similar, with a smoother azimutal pre-stress pressure profile between straight region and heads than for T10.V1.

The T9.V1 and T10.V1 were made similar to T5.V3, but with the first conductor block of the outer layer heads split into two parts, one close to the straight section composed of only two conductors, and the other part with the rest.

The T11.V1 magnet was similar to T9.V1 and T10.V1, made with the same coil geometry and the same collars. The specific feature was that the two innermost conductor blocks of the coil heads of both inner and outer layers were not filled by resin as was done on the reference magnets. This operation fills in fact the voids between adjacent conductors. The hypothesis behind this change was that the filling resin, by microscopic cracking or just big mechanical hysteresis, could be responsible for training quenches. The coils were however built compacted by adding resin fiber-glass pre-preg strips between cable turns in these conductor blocks. Laboratory tests proved that the resulting coil heads were very rigid, deforming by less than 10 microns when compressed longitudinally with a force of about $10 \mathrm{kN}$.

\section{TRAining Performance}

The training behavior of the recent short dipoles (from T4.V6 to T11.V1) is shown in Fig. 1. The training curves of T4.V4 and T5.V3 are presented as reference. As we can see in Fig. 1, all the models exceeded the nominal field for LHC, but only T10.V1 \& V2 had the first quench above $8.4 \mathrm{~T}$. For all these models (except T5.V3 and T4.V4) the training was slow with an average increase of $40 \mathrm{mT}$ per quench. The ultimate field of $9 \mathrm{~T}$ was reached after a minimum of 11 quenches in the best case and the short sample limit was never reached after 15 quenches, far from the good results obtained for the two reference magnets.

The level of the first quenches after the thermal cycle, i.e., the quality of the memory effect is an important parameter for the LHC magnets and statistics are needed in order to predict the behavior of the magnets after a warm up. For this reason the power test campaigns of the short dipoles were mostly carried out in two runs separated by a thermal cycle from $1.9 \mathrm{~K}$ to room temperature and back to $1.9 \mathrm{~K}$. Fig. 2 shows the performance of three short dipole models (T4.V4, T5.V3, and T9.V1) during the first and the second run. The T9.V1 shows memory effect similar to that observed for the first twin-aperture short dipole models T4 and T5 [6]. The magnetic field at first quench increased by about $0.75 \mathrm{~T}$ and corresponds to the value reached after five quenches in the first run. Only the first part of the training curve is memorized consistent with past experience on short and long magnets.

\section{QUENCH LOCALIZATION}

The first double-aperture short dipole models showed that the quench performance at the beginning of the training was limited by weakness of the coil ends [6]. The recent short models tested confirm this trend since no quench below $9 \mathrm{~T}$ occurred in the straight part of the coils. All the quenches below $9 \mathrm{~T}$ were localized in the coil ends, either in the bending part of the ends or in the transition region between the straight and the bending part

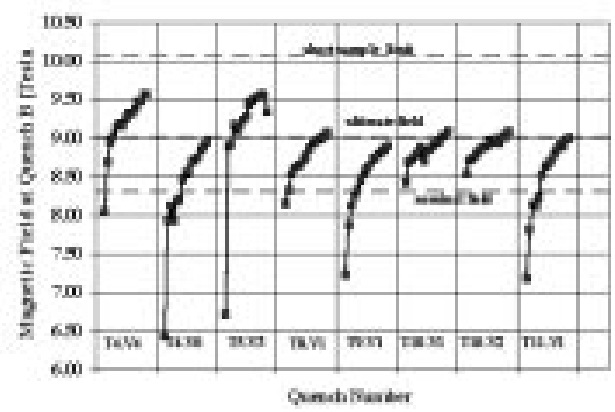

Fig. 1. First 15 training quenches at $1.9 \mathrm{~K}$ of selected short dipole models.

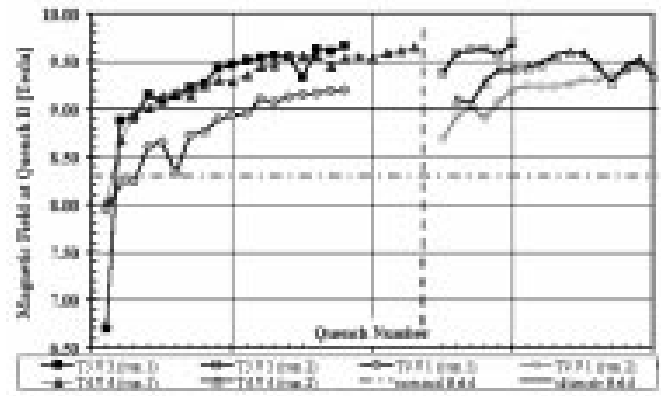

Fig. 2. Training performance of the T9.V1, T4.V4, and T5.V3 during the first run and the second run after a thermal cycle.

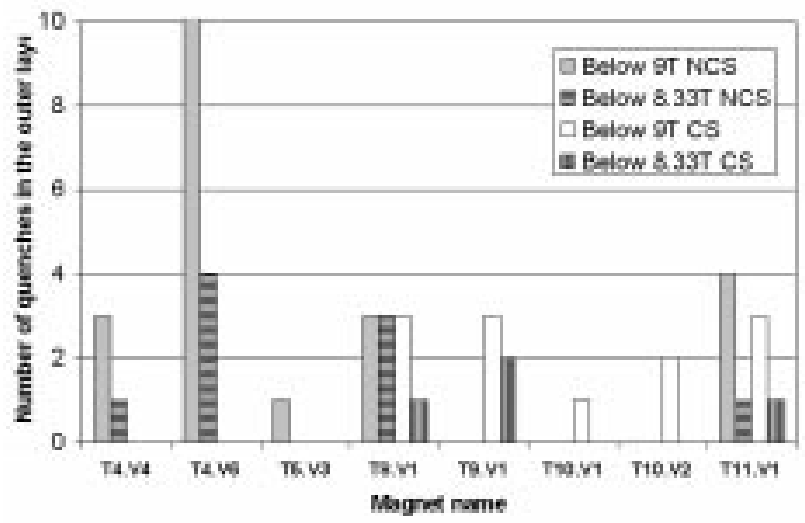

Fig. 3. Number of quenches below $9 \mathrm{~T}$ and $8.33 \mathrm{~T}$ in the outer layer ends, either in the connection (CS) or in the nonconnection ends (NCS).

of the coil. A summary of the localization of quench origins for magnetic field below $8.33 \mathrm{~T}$ (nominal field) and $9 \mathrm{~T}$ (ultimate field) is given in Figs. 3 and 4 for the outer and inner layers respectively.

From Figs. 3 and 4, three conclusions can be derived:

- The models with an additional end-spacer in the outer layers (T9.V1, T10.V1\&V2, T11.V1) display a change in the distribution of the weak regions with an increase of quench location in the inner layer, and no evident improvement of the training performance of the outer layer compared to the reference magnet, which was however particularly good in this region. In fact we have to recall that for the first twin- aperture models and for most of long prototypes [6], [8], the main quench location were 


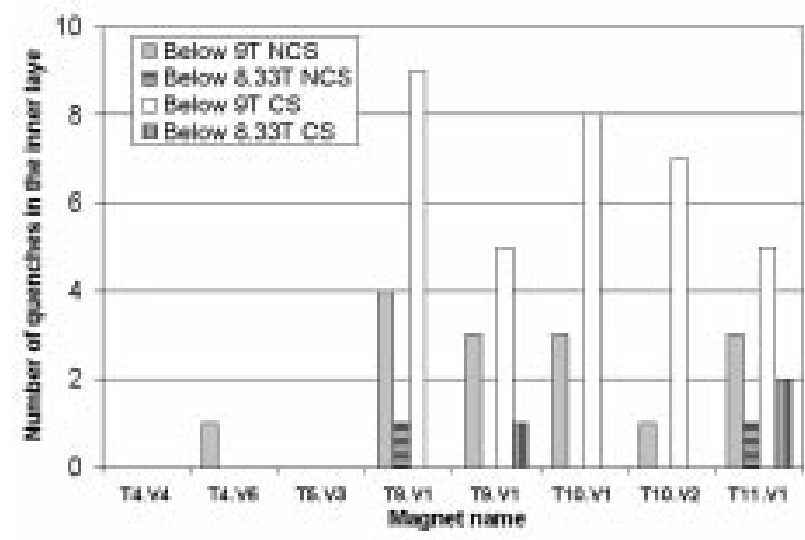

Fig. 4. Same representation as for Fig. 3 but for quenches occurring in the inner layer coil ends.

the outer layer ends. However, over a big magnet production, this additional end-spacer, now implemented on the series magnets, will be maybe advantageous.

- T8.V1 also had many quenches in the inner layer but in this case the training was very slow and 20 quenches were performed before reaching $9 \mathrm{~T}$. The performance of this magnet was clearly limited by the geometrical mismatch introduced during the coil fabrication.

- The quenches in the outer layer were more localized in the nonconnection end (NCS), whereas the connection end (CS) was the main location for quenches in the inner layer. In the case of T4.V6 this imbalance was amplified by the weakness caused by the metallic end-spacers placed in the nonconnection end.

A detailed analysis of the precursors of these quenches showed that most of them were triggered by a mechanical motion of the cable observed, as a spike on voltage taps and on quench antenna signals [9].

\section{CONCLUSION}

The present baseline design of the LHC dipoles provides a good training performance in the straight section of the magnet, where generally first quenches appear above $9 \mathrm{~T}$. The performance below $9 \mathrm{~T}$ is good for the reference magnet T5.V3, which showed a first quench after thermal cycle at about $9.4 \mathrm{~T}$, but still limited by quenches in the coil heads or in the transition regions between straight section and heads. The variants implemented on the last double-aperture models did not provide the key for definitely solving this limitation. However, the work performed allowed to confirm the robustness of the magnet straight section and to better define the direction for next immediate steps.
In particular, it was shown that performance:

- was not better than that of the reference magnet by using more rigid end spacers in the coil heads (with T4.V6);

- does not appear to depend on "as wound" cable block compaction in the coil heads (T11.V1), probably because less compacted heads are filled by resin;

- cannot be easily improved just by splitting cable blocks (T9.V1 and T10.V1 for the outer layer); could be dominated by the geometrical matching in the transition regions (difference between T9.V1 and T10.V1).

At present, the azimuthal pressure profile from the straight section to the coil heads implemented in T10.V1 is being measured with capacitive gauges placed in the magnet mid-plane [10]. The relevant results will be used to build a new version of this magnet with significantly modified pre-stress profile based on a smooth geometrical transition.

Another direction of study which was not explored yet is an important modification of the end-spacer shapes with different angular positioning of the cable.

\section{ACKNOWLEDGMENT}

The authors wish to thank L. Evans for his support and active contribution to the short model activity, and to all the teams involved in magnet fabrication and testing for their excellent work.

\section{REFERENCES}

[1] The LHC Study Group, "The large Hadron collider, conceptual design,", CERN/AC/95-05 (LHC), 1995.

[2] N. Andreev et al., "Performance of five and six block coil geometries in short superconducting dipole models for the LHC," in Proc. PAC-99, New York, 1999.

[3] K. Artoos et al., "Status of the short dipole model program," IEEE Trans. Appl. Superconduct., vol. 10, pp. 49-52, 2000.

[4] N. Andreev et al., "State of the short dipole model program for the LHC," in Proc. EPAC-98, Stockholm, Sweden, 1999, pp. 311-313.

[5] Z. Ang et al., "Coil size and geometric field quality in short model dipoles for LHC," IEEE Trans. Appl. Superconduct., vol. 10, pp. 53-56, 2000

[6] D. Tommasini et al., "Status of the LHC short dipole model programme," in Europ. Phys. Soc., 2000, pp. 2166-2169.

[7] N. Andreev et al., "The 1-m long single-aperture coil test program for LHC," in Proc. EPAC-96, Sitges, Spain, 1996, pp. 2258-2260.

[8] P. Pugnat et al., "Performance of the final dipole prototypes and the first pre-series LHC magnet,", submitted for publication.

[9] B. Dezillie et al., "Study of the mechanical disturbances in superconducting magnets using piezoelectric sensors and quench antenna,", LHC Project Rep. 498, 2001.

[10] N. Siegel, D. Tommasini, and I. Vanenkov, "Design and use of capacitive force transducers for superconducting magnet models for the LHC," in MT-15, Beijing, China, 1997. 